\title{
PROCESAMIENTO ELABORATIVO Y EXPERIENCIA CONSCIENTE EN MEDIDAS IMPLÍCITAS Y EXPLÍCITAS DE MEMORIA EN EL IDIOMA ESPAÑOL
}

\author{
Elaborative processing and conscious experience in implied and explicit memory \\ measures in the spanish lenguage
}

Julieta Andrea Yegros Velázquez ${ }^{1}$

Recibido 15/10/2019

Aceptado 15/12/2019

\begin{abstract}
RESUMEN
En relación a los sistemas de memoria de esta investigación, clasificamos la memoria explícita, que es la voluntaria y consciente; mientras que la memoria implícita no requiere la recuperación intencional o consciente. El objetivo de esta investigación fue determinar el efecto del procesamiento elaborativo completando palabras y apelando al recuerdo por medio de claves. Lo primero pone en manifiesto la intervención de la memoria explicita, mientras lo segundo demuestra la implícita (formas de retención que no van acompañadas de conciencia por parte del sujeto). El diseño ha constado de un grupo experimental, un grupo control y un único post-test. A ambos grupos se les entregó una lista de palabras que a memorizar. Luego se les entregó una hoja con los trigramas, de los cuales algunos correspondían a las palabras memorizadas anteriormente y otras eran distractoras. A un grupo se le entregó el cuestionario de palabras y recuerdo con claves de manera no informada (los sujetos recibieron la instrucción de completar los inicios de las palabras con la primera que se les ocurriese) y al otro grupo se le dio la informada (en la que se les indicó que algunos de los inicios pertenecen a palabras previamente leídas). La hipótesis que se esperaba comprobar era que los participantes de la tarea de memoria que completaron palabras sabiendo que los inicios dados pertenecían a palabras previamente vistas, tendrían mayor éxito en recordarlas durante la ejecución de la prueba, que aquellos que debían escribirlas utilizando solo su memoria implícita. Al finalizar se llegó a la conclusión de que en el grupo experimental recordaron las palabras en menor medida ya que se les pidió que completaran las palabras con lo primero que se les ocurra sin decirle que los inicios son de las palabras que anteriormente habían estudiado. En cambio, en el otro grupo a quienes se les informó que los inicios pertenecían a las palabras vistas anteriormente, se observó una frecuencia mayor en el recuerdo y asociación de palabras mediante la intervención de las letras.
\end{abstract}

Palabras claves: Memoria, palabras, procesamiento elaborativo, frecuencia, recuerdo.

\begin{abstract}
In relation to the memory systems of this research, we classify explicit memory, which is voluntary and conscious; while implicit memory does not require intentional or conscious recovery. The objective of this investigation was to determine the effect of elaborative processing by completing words and recollection by means of clues. The former reveal the intervention of explicit memory, while the latter demonstrate the implicit (forms of retention that are not accompanied by consciousness on the part of the subject). The design consisted of an experimental group, a control group and a post-test only. Both groups were given a list of words that they had to memorize. Then they were handed the sheet with the trigrams, some corresponded to the words memorized previously and others were distracting. One group was given the word questionnaire and recall with keys in an uninformed manner (the subjects were instructed to complete the beginning of the words with the first one that occurred to them) and the other group was given the informed (in which was told that some of the beginnings belong to previously read words). The hypothesis that was expected to be verified was that the participants of the memory task who had to complete words knowing that the given beginnings belong to previously seen words, will have greater success in remembering them during the execution of the test, than those who must write them using only your implicit memory. At the end it was concluded that in the experimental group they remembered the words to a lesser extent since they were asked to complete the words with the first ones that occurred to them without telling them that the beginnings are of the words they had previously studied, in On the other hand, the other group, who were asked to complete the words, were informed
\end{abstract}

${ }^{1}$ Facultad de Filosofía, Universidad Nacional de Asunción. Email: julieyegros@ gmail.com 
that the beginnings belonged to the words seen above, a greater frequency was observed in the memory and association of words through the intervention of the letters.

Keywords: Memory, words, elaborative processing, frequency, memory

\section{Planteamiento del Problema}

El propósito de este experimento fue hacer una investigación en la psicología de la memoria, que ha avanzado monumentalmente a través del tiempo, lográndose demostrar las disociaciones funcionales entre pruebas directas e indirectas de memoria.

Algunos estudios han comprobado el efecto de la atención dividida sobre ambos tipos de memoria, implícita y explícita. Parkin y Russo encontraron que la memoria implícita fue similar en dos condiciones (condición de atención dividida vs. condición de tarea única). Sin embargo, los participantes en la condición de doble tarea actuaron peor en la prueba de recuerdo libre que los de tarea única. Señalaron que las pruebas de priming perceptivo (la exposición a determinados estímulos influye en la respuesta que se da a estímulos presentados con posterioridad) son independientes de la atención.

Como se cita en De la Mata y Sánchez, el investigador Menor Pinilla, quien realizó la investigación que se replicó en este trabajo, con las pruebas informada y no informada, no estaba seguro de si los sujetos de la prueba no informada se daban cuenta por sí solos de la relación entre la lista de palabras de la fase de aprendizaje y los indicios de las palabras que debían completar en la segunda fase, o si se trataba del uso de su memoria implícita. Por ello, el autor empleó el uso de un cuestionario para saber si los sujetos descubrían la relación entre las dos fases de la prueba. De esta manera, Menor Pinilla aborda el tema del papel de la conciencia en la recuperación de la información, tema que está recibiendo cada vez más atención en los últimos años.

La intención de esta investigación ha sido demostrar cómo actúa la memoria semántica durante el proceso de remembranza y asociación de una información almacenada en la memoria a largo plazo. Para lograr dicho acometido hemos planteado la siguiente pregunta ¿Cómo actúan las memorias explícita e implícita al momento del proceso elaborativo durante la ejecución de una tarea de memoria?

\section{Justificación y uso de los resultados}

Como es citado en Morris, la investigación científica sobre la memoria empezó con los experimentos de Hermann Ebbinghaus en el siglo XIX. En la actualidad, el modelo de procesamiento de la información de la memoria describe cómo se codifica, almacena y recupera la información de la memoria.

La información bruta fluye de los sentidos hacia los registros sensoriales, donde se desvanece o se procesa a partir del conocimiento y la información ya existentes. La información que se considera significativa se transfiere a la memoria a corto plazo para su procesamiento ulterior; el resto se desecha (Ayala, 2014). Una vez en la memoria a corto plazo, la información se olvida o se transfiere a la de largo plazo, donde se guarda para ser recuperada cuando se necesite de forma consciente.

Basados en la concepción de la memoria semántica, la cual forma parte de la memoria explícita; y a largo plazo, que guarda los hechos y la información general, cuyos recuerdos abarcan el conocimiento de sucesos históricos, la capacidad para reconocer amigos y conocidos, o la información aprendida en la escuela, como el vocabulario especializado, la lectura, la escritura, entre otros (Ayala, 2014).

Como hemos dicho anteriormente, la investigación en psicología de la memoria ha demostrado disociaciones funcionales entre pruebas directas e indirectas de memoria, en las 
primeras se ponen de manifiesto la intervención de la memoria explícita y en las segundas demuestran formas de retención por parte del sujeto que no van acompañadas de consciencia, que se podría atribuir a la memoria implícita (De la Mata y Sánchez, 1996).

\section{Fundamento teórico}

- "Memoria: capacidad para recordar las cosas que hemos experimentado, imaginado y aprendido", (Morris y Maisto, 2005, p.185).

- "Memoria de corto plazo (MCP): memoria de trabajo; almacena brevemente y procesa la información seleccionada de los registros sensoriales" (Morris y Maisto, 2005, p.189).

- "Memoria de largo plazo (MLP): la parte de la memoria que es más o menos permanente, correspondiente a todo lo que "sabemos" (Morris y Maisto, 2005, p.189).

- "Efecto de posición serial: efecto que consiste en que cuando nos piden recordar una lista de elementos no relacionados, el desempeño es mejor para los elementos colocados al inicio y al final de la lista"' (Morris y Maisto, 2005, p.192).

- "Segmentación: El agrupamiento de la información en unidades significativas para que la memoria de corto plazo la maneje de manera más sencilla" (Morris y Maisto, 2005, p.190).

- "Repaso mecánico: Consiste en retener la información en la memoria repitiéndola una y otra vez'” (Morris y Maisto, 2005, p.191).

- "Repaso elaborativo: Conexión de la nueva información en la memoria de corto plazo con el material familiar almacenado en la memoria de largo plazo"' (Morris y Maisto, 2005, p.193).

- "Esquema: Conjunto de creencias o expectativas acerca de algo que se basan en la experiencia previa"' (Morris y Maisto, 2005, p.194).

- "Memoria episódica: Parte de la memoria de largo plazo que almacena eventos experimentados personalmente.

- Memoria semántica: Parte de la memoria de largo plazo que almacena hechos e información general.

- Memoria procedimental: Parte de la memoria de largo plazo que almacena información relacionada con habilidades, hábitos y otras tareas perceptualesmotoras", (Morris y Maisto, 2005, p.195).

- "La memoria emocional: comprende respuestas emocionales aprendidas a varios estímulos: todos nuestros amores y odios, nuestros temores racionales e irracionales, nuestros sentimientos de disgusto y ansiedad.

- Memoria explícita: Memoria para información que podemos expresar fácilmente en palabras y que estamos conscientes de tener; esos recuerdos pueden recuperarse de la memoria de manera intencional.

- Memoria implícita: Memoria para la información que no podemos expresar fácilmente en palabras y que podemos no estar conscientes de tener; esos recuerdos no pueden recuperarse intencionalmente de la memoria'" (Morris y Maisto, 2005, p.196).

\section{Memoria explícita e implícita}

Los psicólogos distinguen entre cosas que conocemos conscientemente y que podemos describir fácilmente, como la memoria episódica y semántica (memoria explícita), y las cosas que no concemos conscientemente y que tampoco podemos describir con facilidad, como la memoria procedimental y emocional (memoria implícita). 
El interés serio en la distinción entre la memoria explícita e implícita surgió como resultado de experimentos realizados con personas que habían sufrido daño encefálico que, como se pensaba, les impedía formar nuevos recuerdos de largo plazo.

Brenda Milner (como se citó en Morris y Maisto, 2005) estudió el ahora famoso caso del paciente H. M., un joven al que se le extirpó una parte del encéfalo para controlar los graves ataques epilépticos que padecía.

La cirugía redujo considerablemente la frecuencia y severidad de los ataques, pero lo dejó con un nuevo problema. Al parecer, $\mathrm{H}$. M. no podía formar nuevos recuerdos. Podían presentarle a alguien una y otra vez, y siempre era como si acabara de conocer a la persona. Podía leer la misma revista día tras día y no recordar que la había visto antes. Los viejos recuerdos estaban intactos: recordaba cosas que había aprendido mucho antes de la operación, pero no podía aprender nada nuevo.

Un día, Milner le pidió a H. M. que dibujara una estrella mientras miraba en un espejo. Esta simple tarea es sorprendentemente difícil, pero, con cierta práctica, la mayoría de la gente muestra un progreso constante. Sorprendentemente así lo hizo H. M. Cada día dibujaba mejor la estrella, como lo haría una persona sin daño encefálico, pero no recordaba siquiera haber intentado la tarea. El desempeño de H. M. demostraba que todavía podía aprender una habilidad, pero no tenía memoria de haberlo hecho.

La investigación sobre la memoria implícita demuestra que podemos almacenar experiencias emocionales, como el trauma físico y psicológico, y que esos recuerdos pueden afectar nuestra conducta años más tarde aun cuando no tengamos recuerdo consciente de esas experiencias.

\section{Preparación}

La investigación sobre un fenómeno llamado preparación también demuestra la distinción entre la memoria explícita e implícita. Por ejemplo, a alguien se le muestra una lista de palabras en inglés que incluye la palabra tour sin pedirle que recuerde ninguna de las palabras. Más tarde, se le muestra una lista de fragmentos de palabras, incluyendo 'ou', y se le pide que llene los espacios en blanco para formar una palabra.

En esas circunstancias, es mucho más probable que escriba tour a que escriba four, pour o sour, opciones que son tan aceptables como tour. Aunque no se le haya pedido recordar la palabra tour, el simple hecho de ser expuesto a ella lo prepara para escribirla.

Un hecho interesante es que la gente con amnesia se desempeña tan bien como la gente con memoria normal en las tareas de preparación. Por ejemplo, un estudio (Warrington y Weiskrantz, 1970 citado en Morris y Maisto, 2005) presentó a varias personas con amnesia una lista de palabras para recordar. Cuando se pidió a esos pacientes que recordaran las palabras o que las eligieran de listas más largas, tuvieron un pobre desempeño, como era de esperarse. Pero cuando los experimentadores mostraron a los pacientes fragmentos de las palabras y les pidieron conjeturar de qué palabra se trataba o que dijeran lo primero que les viniera a la cabeza, mencionaron casi tantas palabras de la lista como la gente que no sufría de amnesia, dando cuenta de que las víctimas de amnesia tenían recuerdos implícitos perfectamente claros de palabras de las que no tenian consciencia de haber escuchado explícitamente.

\section{El fenómeno de la punta de la lengua}

Todos hemos tenido la experiencia de saber una palabra y no ser capaces de recordarla. A esto se denomina el fenómeno de la punta de la lengua según Brown y McNeil (citado en Morris y Maisto, 2005). 
Dicen White y Abrams (citado en Morris y Maisto, 2005) que, aunque todos experimentamos el fenómeno de la punta de la lengua, esas experiencias se hacen más frecuentes en situaciones estresantes y a medida que la gente envejece.

Más aún, mientras se experimenta el fenómeno de la punta de la lengua vienen a la mente otras palabras (por lo regular con un sonido o significado similar al de la palabra que está buscando) y esas palabras a menudo interfieren y sabotean su intento de recordar la palabra deseada. Cuanto más se esfuerce, peor se vuelve el fenómeno de la punta de la lengua. La mejor manera de recordar una palabra bloqueada es dejar de intentar recordarla. Según Schwartz (como se cita en Morris y Maisto, 2005) la mayoría de las veces, la palabra que buscaba le vendrá a la cabeza minutos o incluso horas después de que dejó de buscarla conscientemente.

La distinción entre las memorias explícita e implícita significa que algún conocimiento es literalmente inconsciente. Más aún, las memorias explícita e implícita también parecen relacionarse con estructuras y trayectorias nerviosas diferentes. Sin embargo, las memorias por lo regular trabajan juntas. Cuando recordamos haber ido a un restaurante chino, no sólo recordamos cuándo y dónde comimos y con quién estábamos (memoria episódica), sino también la naturaleza de la comida que ordenamos (memoria semántica), las habilidades que aprendimos como comer con palillos (memoria procedimental) y la vergüenza que sentimos al derramar el té (memoria emocional).

Cuando recordamos los eventos, por lo general, no experimentamos esos tipos de memorias como distintas y separadas; más bien están integralmente conectadas, como lo estuvieron en las experiencias originales. El que sigamos recordando las experiencias con precisión en el futuro depende en gran medida de lo que suceda en nuestro encéfalo.

\section{La formación de los recuerdos}

Según Squire y Kandel (citado en Morris y Maisto, 2005) todo lo que aprendemos se registra finalmente en el encéfalo en la forma de cambios en el tamaño, forma, funcionamiento químico y conexión entre neuronas. Cuando aprendemos cosas nuevas se forman nuevas conexiones en el encéfalo; cuando repasamos o practicamos las cosas aprendidas previamente, se fortalecen las antiguas conexiones. Esos cambios químicos y estructurales pueden continuar por un periodo de meses o años, durante los cuales se incrementa el número de conexiones entre neuronas, al igual que la probabilidad de que las células se exciten una a la otra a través de descargas eléctricas, un proceso conocido como potenciación a largo plazo (PLP).

Si bien el aprendizaje tiene lugar en el encéfalo, también recibe influencia de eventos que ocurren en otras partes del cuerpo. En particular, hay dos hormonas, la epinefrina y el cortisol, que afectan la retención a largo plazo. McGaugh (se citó en Morris y Maisto, 2005) menciona varios estudios realizados con ratas, monos y seres humanos han demostrado, por ejemplo, que la epinefrina puede mejorar el recuerdo de la exposición a estímulos asociados con experiencias desagradables.

Durante mucho tiempo se ha sabido que el encéfalo tiene áreas especializadas para la visión y la audición, por lo que parece lógico que una parte del encéfalo pueda estar dedicada a la memoria.

Con la esperanza de localizar el sitio específico de la memoria, Lashley (1950) extirpó sistemáticamente diversas partes del encéfalo de las ratas después de que habían aprendido una tarea. Aunque la pérdida de parte del encéfalo debilitó los recuerdos, no los eliminó por completo. De hecho, el desempeño tuvo menos que ver con el área del encéfalo extirpada que con la cantidad de tejido extraído. Cuanto más tejido extirpara 
Lashley del encéfalo, menos recordaban las ratas. Lashley se vio obligado a concluir que los recuerdos se almacenan en todo el encéfalo.

Aunque no todos los recuerdos se almacenan en un lugar, esto no significa que los recuerdos se distribuyan al azar por todo el encéfalo. De hecho, la investigación de Rolls (2000) ha proporcionado amplia evidencia de que diferentes partes del encéfalo se especializan en el almacenamiento de ciertos recuerdos. Por ejemplo, según los recuerdos de corto plazo parecen localizarse principalmente en la corteza prefrontal y el lóbulo temporal. La memoria semántica de largo plazo parece localizarse sobre todo en los lóbulos frontal y temporal de la corteza, los cuales, de manera interesante, también parecen desempeñar un papel destacado en la conciencia. Por ejemplo, la investigación muestra un incremento en la actividad de un área particular del lóbulo temporal izquierdo cuando se pide a la gente que recuerde los nombres de personas. Damasio, Grabowski, Tranel, Hichawa y Damasio (citado en Morris y Maisto, 2005) mencionan que un área cercana muestra mayor actividad cuando se le pide que recuerde los nombres de animales, y otra área vecina se activa cuando se le pide que recuerde los nombres de herramientas. La destrucción de esas áreas de la corteza (como consecuencia de daño encefálico, cirugía, apoplejía o enfermedad) deriva en una pérdida selectiva de la memoria. Algunos pacientes son incapaces de recordar el nombre de una herramienta, aunque pueden describir cómo se usa, mientras que otros son incapaces de recordar el nombre de un viejo amigo o de su cónyuge.

La memoria episódica también encuentra su morada en los lóbulos frontal y temporal. Pero alguna evidencia muestra que las memorias episódica y semántica están relacionadas con partes diferentes de esas estructuras encefálicas. Wood y sus colegas (se cita en Morris y Maisto, 2005) compararon el flujo sanguíneo en el encéfalo mientras las personas trabajaban en dos diferentes tipos de tareas (el flujo sanguíneo en un área se asocia con la actividad en la misma). Algunas personas realizaban una tarea relacionada con la memoria episódica, mientras que otras efectuaban una tarea relacionada con la memoria semántica. Los investigadores encontraron que los dos tipos de tareas produjeron un incremento en el flujo sanguíneo en áreas diferentes del encéfalo.

La memoria procedimental parece localizarse de manera principal en el cerebelo (un área requerida para el equilibrio y la coordinación motora) y en la corteza motora. Grafton (citado en Morris y Maisto, 2005) menciona que cuando las personas desempeñan una tarea que requiere que sigan un objeto en rotación con un punzón sostenido en la mano, se incrementa la actividad en la corteza motora.

Las estructuras subcorticales también participan en la memoria de largo plazo. Por ejemplo, el hipocampo se ha vinculado con el funcionamiento de la memoria episódica (Rolls, 2000), así como con la capacidad para recordar relaciones espaciales. La memoria emocional depende de la amígdala, una estructura que yace cerca del hipocampo.

Dicen Pare, Collins y Guillaume (citado en Morris y Maisto, 2005) que la amígdala parece jugar un papel en la memoria emocional que es similar al papel que desempeña el hipocampo en la memoria episódica, semántica y procedimental. Por ejemplo, el daño en la amígdala reduce la capacidad para recordar nuevas experiencias emocionales, pero no impide el recuerdo de eventos emocionales que ocurrieron antes del daño, aunque a menudo se recuerdan como hechos neutrales, es decir, vacíos de contenido emocional.

Esto explica por qué los individuos con lesiones en la amígdala a menudo son incapaces de "interpretar" las expresiones faciales aun cuando reconocen el rostro de la persona. 


\section{Objetivos de investigación}

\section{General}

- Analizar cómo actúan la memoria explícita e implícita en el procesamiento elaborativo durante la realización de una tarea de memoria.

\section{Específicos}

- Describir las posibles asociaciones entre ambos tipos de memoria (implícita/explícita) durante el estado consciente.

- Comprender el funcionamiento de la memoria semántica y sus implicancias al momento de recordar palabras simples.

- Comparar los resultados obtenidos durante la aplicación de la prueba T demostrando si se aprueba o rechaza la hipótesis propuesta.

\section{Hipótesis}

- Los participantes de una tarea de memoria que tuvieren que completar palabras sabiendo que los inicios dados pertenecen a palabras previamente vistas, tendrán mayor éxito en recordarlas durante la ejecución de la prueba, que aquellos que deben escribirlas utilizando solo su memoria implícita.

\section{Metodología de la investigación}

\section{Tipo de estudio y diseño en general}

Ha sido de naturaleza experimental, pues se buscó descubrir la influencia de la variable independiente sobre la variable dependiente.

El diseño es experimental puro, donde se conformaron dos grupos de sujetos asignados aleatoriamente, en un grupo control y otro experimental, a los cuáles se aplicó el instrumento de medición.

Es de corte transversal, la población de 70 individuos de los cuales fueron seleccionados en forma aleatorizada 40 sujetos para la muestra, de ambos sexos pertenecientes al segundo curso de la primera sección del turno noche de la carrera de psicología del área básica de la Facultad de Filosofía (Casa Central) del barrio Ita Pyta Punta de Asunción.

Las condiciones de codificación (juicio semántico y juicio preceptivo), tarea de memoria (completar palabras versus recuerdo con claves) e instrucciones (prueba NO INFORMADA, en la que los sujetos recibieron la instrucción de completar los inicios de la palabra con la primera que se les ocurra, y prueba INFORMADA, en la que se les indicó que algunos de los inicios pertenecen a palabras previamente estudiadas, aunque deberían haberlas completado con las primeras que se les ocurriera). Esta última variable afecta, únicamente, a la tarea de completar palabras.

\section{Operacionalización de las variables}

En un experimento que implica la manipulación de memoria implícita y explícita en tareas de memoria, donde el propósito es medir la memoria semántica, se conformaron dos grupos: un grupo experimental y un grupo control. Una vez formados ambos grupos en forma aleatoria, se procedió con las instrucciones, la prueba no informada se le dio al grupo experimental, los sujetos recibieron la instrucción de completar los inicios de las palabras con la primera que se les ocurra, y la prueba informada, al grupo control, en la que se les indicó 
que algunos de los inicios pertenecen a palabras previamente estudiadas, aunque deberían haberlas completado con las primeras que se les ocurriera. Una vez realizada la tarea de completar palabras, el grupo experimental (con la prueba no informada) respondieron un cuestionario para evaluar hasta qué punto se han dado cuenta de que estaban completando los inicios de las palabras con otras previamente estudiadas.

- Variables Dependientes: Memoria Explícita e Implícita las cuales se busca medir a través del recuerdo de palabras.

- Variable Independiente: Tarea de memoria, la cual es manipulada para medir la memoria explícita e implícita de los sujetos.

- Variable activa: Tarea de memoria la cual es manipulada constantemente a través de la misión de recordar y completar palabras a través de trigramas.

- Participantes

El experimento se replica aplicándolo a 40 alumnos de la carrera de Psicología de segundo curso de la primera sección del turno noche de la Facultad de Filosofía UNA (Casa Central) del Barrio Ita Pyta Punta de Asunción.

\section{Procedimientos}

\section{Técnicas de recolección de datos}

Durante esta investigación se procedió a replicar uno de los experimentos realizados por Menor Pinilla (L. de la Mata \& Sánchez, 1996, p. 35) donde analizó el efecto del procesamiento elaborativo en dos pruebas en su formato y material.

Se seleccionaron 40 sujetos de entre 19 y 30 años de ambos sexos en forma aleatoria. El nivel de estudios es semejante de modo que todos se encuentren cursando el segundo curso de la carrera de psicología del área básica.

El primer paso para el éxito de toda investigación científica es acudir a la revisión bibliográfica, recurrir a las fuentes e indagar los escritos previos que hagan referencia al tema investigado, para hacer la construcción del marco teórico.

La prueba puede administrarse de dos formas: colectivamente, mediante un ordenador con el software adecuado y de manera individual, aplicada por los experimentadores, con un plan elaborado por ellos. Consta de dos fases: aprendizaje y prueba de memoria.

Una vez formados los grupos experimental y de control, se procede con las instrucciones (prueba NO INFORMADA, en la que los sujetos reciben la instrucción de completar los inicios de las palabras con la primera que se les ocurra, y prueba INFORMADA, en la que se les indica que algunos de los inicios pertenecen a palabras previamente estudiadas, aunque deben completarlas con las primeras que se les ocurra). Esta última variable afecta, únicamente, a la tarea de completar palabras.

Una vez realizada la tarea de completar palabras, todos los sujetos de la condición de PRUEBA NO INFOMADA responden un cuestionario para evaluar hasta qué punto se han dado cuenta de que estaban completando los inicios de las palabras con otras previamente estudiadas (De la Mata y Sánchez, 1996, p.37).

\section{Materiales}

Se emplearon los siguientes materiales:

1. Dos listas de codificación compuestas por 20 palabras cada una, lista I y lista II, tomadas de Menor Pinilla (1993). Las palabras han sido elegidas de modo que tengan una 
frecuencia de uso media y que no aparezca más de una palabra en la lista con el mismo comienzo. Ambas listas se emplearon en las distintas condiciones experimentales.

2. En la tarea de completar palabras se emplearon los inicios de las 40 palabras de las listas I y II, de modo que cada sujeto completa los inicios de 20 palabras que le han sido presentadas en la fase de codificación y 20 que no (distractores).

3. Un cuestionario compuesto por cuatro preguntas destinadas a evaluar la conciencia de los sujetos de la condición de prueba no informada durante la realización de la tarea de completar palabras. El cuestionario ha sido tomado de Bowers y Schacter (1990) y está compuesto por cuatro preguntas. Las dos primeras preguntas tienen una respuesta abierta ('¿Cual crees tú que era el objetivo de la tarea de completar palabras?'). "Describe la estrategia que has usado para completar las palabras en esa tarea"). Las otras dos exigen una respuesta más precisa (¿"Has notado alguna relación entra las palabras que viste en las tarjetas y las que has completado en la tarea anterior?". "Mientras completabas las palabras, ¿notaste si lo hacías con palabras de las que habías visto antes en las tarjetas?") (De la Mata y Sánchez, 1996, p.37).

\section{Aspectos éticos}

Antes de comenzar el procedimiento con previo consentimiento de los voluntarios, se procedió a mencionar o relatar cuáles eran los aspectos principales como que la aplicación de instrumentos es de carácter anónimo sin la solicitud de ninguna información personal.

Según Kerlinger todas las personas involucradas en un proyecto de investigación están limitadas por los códigos de ética sin importar si son o no profesionales de la psicología o miembros de la Asociación Americana de Psicología (APA). (Kerlinger, 2002).

Así mismo Morris menciona que obligatoriamente las personas que participen de un experimento deben estar informadas de tal suceso, explicando que las normas éticas establecidas por la APA (Morris, 2001) incluyen criterios para la planificación de la investigación, protección de los participantes, confidencialidad, desengaño, engaño, consentimiento informado y libertad de coerción estipulando cuanto a continuación dice:

- Los participantes deben ser informados de la naturaleza de la investigación en un lenguaje fácil de comprender.

- Se debe documentar el consentimiento informado.

- Se deben explicar de antemano los riesgos, los posibles efectos adversos y las limitaciones de la confidencialidad.

- Si la participación es una condición para obtener los créditos en un curso deben ofrecerse actividades alternativas equitativas.

- No se debe engañar a los participantes acerca de los aspectos de la investigación que pudiera afectar su disposición de participar, como los riesgos o experiencias emocionales desagradables.

- El engaño acerca de las metas de la investigación sólo se puede utilizar cuando sea absolutamente necesario para la integridad del estudio (Morris, 2001)

\section{Análisis y discusión de los resultados}

La muestra está compuesta por 40 alumnos, de la carrera de psicología del 2do curso 1ra sección, integrada por adultos varones y mujeres del turno noche con edades comprendidas 
de 20 a 30 años de la Facultad de Filosofía UNA (Casa Central) del Barrio ItaPyta Punta de Asunción.

La cantidad de varones encuestados fue de 15 y el número de mujeres fue de 25 , de un total de 40 personas de la muestra. Esto hizo una diferencia de participantes del sexo masculino un $37,5 \%$ y un $62,5 \%$ del sexo femenino.

Tabla 1. Cantidad de personas pertenecientes a la muestra con sus edades respectivas

\begin{tabular}{|l|l|}
\hline Edad & Cantidad de Personas \\
\hline 19 & 7 \\
\hline 20 & 10 \\
\hline 21 & 13 \\
\hline 23 & 5 \\
\hline 26 & 2 \\
\hline 30 & 3 \\
\hline TOTAL & $\mathbf{4 0}$ \\
\hline
\end{tabular}

De un total de la muestra de 40 sujetos, los participantes de mayor edad tienen 30 años mientras que los de menor edad oscilan entre los 19 años.

La edad promedio de la muestra está entre los 23 años.

Tabla 2. Aplicación de la prueba estadística de T Student

\begin{tabular}{|c|c|c|c|c|c|c|}
\hline $\begin{array}{l}\mathrm{N}^{\circ} \quad \text { de } \\
\text { Sujeto }\end{array}$ & $\begin{array}{l}\text { A1 palabras } \\
\text { recordadas }\end{array}$ & $\mathrm{XX}$ & $(\mathrm{XX})$ & $\begin{array}{l}\text { A2 palabras } \\
\text { recordadas }\end{array}$ & XX & $(\mathrm{XX})$ \\
\hline 1 & 10 & 2 & 4 & 20 & 4 & 16 \\
\hline 2 & 12 & 0 & 0 & 20 & 4 & 16 \\
\hline 3 & 14 & 2 & 4 & 19 & 3 & 9 \\
\hline 4 & 18 & 6 & 36 & 15 & $\ldots 1$ & 1 \\
\hline 5 & 20 & 8 & 64 & 17 & 1 & 1 \\
\hline 6 & 19 & 7 & 49 & 9 & $\_7$ & 49 \\
\hline 7 & 8 & -4 & 16 & 7 & -9 & 81 \\
\hline 8 & 4 & -8 & 64 & 15 & -1 & 1 \\
\hline 9 & 16 & 4 & 16 & 12 & -4 & 16 \\
\hline 10 & 11 & -1 & 1 & 13 & -3 & 9 \\
\hline 11 & 11 & -1 & 1 & 14 & 2 & 4 \\
\hline 12 & 6 & -6 & 36 & 17 & 1 & 1 \\
\hline 13 & 13 & 1 & 0 & 20 & 4 & 16 \\
\hline 14 & 12 & 0 & 1 & 20 & 4 & 16 \\
\hline 15 & 17 & 5 & 0 & 19 & 3 & 9 \\
\hline 16 & 20 & 8 & 25 & 18 & 2 & 4 \\
\hline 17 & 14 & 2 & 64 & 18 & 2 & 4 \\
\hline 18 & 3 & _9 & 4 & 17 & 1 & 1 \\
\hline 19 & 6 & _6 & 81 & 16 & 0 & 0 \\
\hline 20 & 9 & -3 & 36 & 20 & 4 & 16 \\
\hline
\end{tabular}

Tabla 2. Se muestra el número de palabras recordadas según el orden de los sujetos y grupo al que pertenecen, así también como el promedio de los datos recolectados

En la tabla 2 se trascribieron los datos recolectados a través de los instrumentos y posteriormente analizados mediante el procedimiento estadístico de la prueba $T$ Student.

Se agrupó a los sujetos de la muestra en los Grupos A1 (EXPERIMENTAL) y el A2 (CONTROL), para conocer los promedios respectivos de cada grupo y luego aplicarlos a las fórmulas para hallar los datos de la desviación estándar, grado de libertad, nivel de significancia, entre otros. 
Tabla 3. Se proponen los resultados encontrados tras la aplicación de las ecuaciones para hallar la T de Student.

\begin{tabular}{|l|l|l|l|l|l|l|l|l|l|l|l|l|}
\hline N1 & N2 & $\mathrm{m} 1$ & $\mathrm{~m} 2$ & $\begin{array}{l}\text { EEm } \\
1\end{array}$ & EEm2 & $\begin{array}{l}\text { EEm } \\
1 \mathrm{~m} 2\end{array}$ & $\mathrm{~T}$ & De1 & De2 & $\begin{array}{l}\text { Grado } \\
\text { de } \\
\text { Libertad }\end{array}$ & $\begin{array}{l}\text { Nivel de } \\
\text { Significanci } \\
\text { ar entre } \\
0,01 \% \text { y } \\
0,05 \%\end{array}$ & $\begin{array}{l}\text { Punto } \\
\text { Crítico }\end{array}$ \\
\hline 20 & 20 & 11,6 & 16,3 & 1,16 & 0,82 & 1,42 & 2,8 & 5,2 & 3,7 & 38 & $\begin{array}{l}\text { Se encuentra } \\
\text { entre el 2,4 } \\
\text { y } 1,68\end{array}$ & 1,68 \\
\hline
\end{tabular}

- Posteriormente agrupados los datos en la tabla 2 de frecuencias se pasó a analizar los, donde se encontraron los siguientes resultados:

- Tanto las muestras de G1 como G2 eran de 20 sujetos, cada uno de ellos escogidos aleatoriamente.

- Los promedios hallados entre N1 y la sumatoria total de los datos acumulados entre los 20 sujetos, dio como resultado 11,6; unidades y el promedio del G2 fue de 16,3 unidades.

- Los errores encontrados fueron de 1,16 para el G1 y de 0,82 para el G2. Así como el error promedio total entre ambos grupos fue de 1,42 unidades.

- La prueba de T arrojó un resultado negativo de 2,8 unidades.

- El hallazgo de las desviaciones estándar fue de 5,2 unidades para De1 y para De2 de 3,7 .

- El grado de libertad encontrado fue de 38 unidades, encontrándose en un nivel de significancia del 5\% entre los datos de 2,4 y 1,68 unidades.

- Finalmente, el punto crítico encontrado fue de 1,68 unidades.

\section{Discusión}

Respecto a las variables que se utilizaron en esta investigación, mencionaban De la Mata y Sánchez (1996) que hay diferencias notables en los resultados obtenidos en las pruebas de memoria explícitas (recuerdo y reconocimiento), depende de factores como el proceso elaborativo. Así las condiciones de codificación que favorecen el análisis del significado de los estímulos presentados dan lugar a mejores resultados que aquellos que promueven el procesamiento de aspectos estructurales o perceptivos del estímulo.

Autores como Graf y Mandler (1984), citados en De la Mata y Sánchez, señalaron que el efecto del procesamiento elaborativo no se manifiesta al emplear medidas indirectas, en estas pruebas indirectas podemos incluir la de 'completar palabras', como la que se replica en este trabajo, se les da a los sujetos las tres primeras letras de una serie de palabras, pidiéndoles que completen hasta formar las palabras que ellos quieran. A pesar de que no se les pide o da indicaciones explícitas, los sujetos tienden a formar palabras previamente presentadas, lo que pondría en manifiesto una forma de retención implícita, es decir, no consciente. En este dato nos hemos basado para obtener nuestra hipótesis.

En cambio, Menor Pinilla (1993) citado en De la Mata y Sánchez, ha obtenido resultados diferentes a los de Graf y Mandler. Los resultados de su prueba de completar palabras sí se han visto favorecidos por el análisis semántico de las palabras en la fase de aprendizaje, dando lugar a una mejor retención que los análisis centrados en la estructura ortográfica de las palabras.

Menor Pinilla agregó una variable extra a su experimento, que es la de la prueba informada en la que los sujetos reciben la instrucción de completar los inicios de las palabras 
con la primera que se les ocurriese, y la prueba no informada en la que se les indica que algunos de los inicios pertenecen a palabras previamente leídas. Con esto se llegó a unas posibles explicaciones por parte de Bowers y Schacter (1990) citado en De la Mata y Sánchez, sobre el efecto del procesamiento elaborativo en la tarea de completar palabras, y es que a pesar de que los sujetos no recibían las instrucciones de manera explícita, es decir no se les decía que las claves hacían referencia a las palabras presentadas anteriormente, los sujetos igual podían darse cuenta de manera espontánea de que las claves eran de las mismas palabras.

Es citado en De la Mata y Sánchez que Menor Pinilla empleó un cuestionario desarrollado por Bowers y Schacter, con el que se intenta determinar si los sujetos con la condición de la prueba no informada llegaban a descubrir la relación entre las dos pruebas y convertían la tarea en una prueba explícita de memoria.

Con los datos expuestos anteriormente, es que desarrollamos nuestra hipótesis que consiste en que los participantes de una tarea de memoria que tuvieren que completar palabras sabiendo que los inicios dados pertenecen a palabras previamente vistas, tendrán mayor éxito en recordarlas durante la ejecución de la prueba, que aquellos que deben escribirlas utilizando solo su memoria implícita.

Después de haber aplicado el test, recolectado y analizado los datos, podemos confirmar nuestra hipótesis, ya que los datos muestran que claramente, los sujetos informados de la prueba obtuvieron mejor rendimiento a la hora de completar las palabras en comparación a los que sólo utilizaron su memoria implícita.

\section{Conclusión}

Según el análisis e interpretación de los datos y fuentes recolectados, se llegó a las siguientes conclusiones:

- Como pudimos corroborar, los conceptos de memoria y aprendizaje están estrechamente relacionados. Mientras el aprendizaje es un proceso mediante el cual adquirimos información y experiencia, la memoria se refiere a la persistencia de lo aprendido en un estado que puede ser evocado posteriormente. En este sentido, la memoria es el resultado del aprendizaje.

- Tanto los objetivos generales como los específicos de la propuesta investigativa, se cumplieron durante todo el proceso investigativo.

- El objetivo genérico de: Analizar cómo actúan la memoria explícita e implícita en el procesamiento elaborativo durante la realización de una tarea de memoria, se cumplió a cabalidad y los resultados han sido demostrados estadísticamente.

- En cuanto a los objetivos específicos, se pueden notar los resultados en la tabulación y posterior análisis de los mismos.

- Las posibles asociaciones entre ambos tipos de memoria (implícita/explícita) durante el estado consciente, han sido descritas en su complejidad dentro del cuerpo teórico de la investigación y llevada a la realidad durante la intervención del experimento tanto en el grupo experimental como en el grupo control, donde se destaca el funcionamiento de la memoria semántica y sus implicancias al momento de recordar palabras simples.

- Comparar los resultados obtenidos durante la aplicación de la prueba T demostrando si se aprueba o rechaza la hipótesis propuesta. 
- La media entre las edades de los sujetos de ambos grupos, control y experimental, ronda una media de 23 años.

- Hubo mayor predominancia de individuos del sexo femenino con un porcentaje de $62,5 \%$ en cambio del sexo masculino fue un mínimo de $37,5 \%$.

- El resultado de la prueba T con un valor de 2,78 unidades; resultó superior a los niveles de significancia entre $0,01 \%$ y $0.05 \%$ así como también al punto crítico, por lo tanto, la hipótesis queda demostrada de forma estadística que se cumple en su totalidad, siendo rechazada una posible hipótesis nula, y la diferencia se puede encontrar al azar en más del $5 \%$.

- El Grado de Libertad obtenido de la suma de los promedios de ambos grupos menos 2, se calculó con la finalidad de estimar los valores de parámetros de población desconocidos y calcular la variabilidad de esas estimaciones, así como también para caracterizar una distribución específica, tuvo un resultado de 38 unidades.

- En la tabla 2 de la prueba estadística del T Student se observó que en el grupo experimental recordaron las palabras en menor medida ya que se les pidió que completaran las palabras con las primeras que se les ocurra sin decirle que los inicios son de las palabras que anteriormente habían estudiado, en cambio el otro grupo, a quienes se les pidió que completaran las palabras, se les informó que los inicios pertenecían a las palabras vistas anteriormente, se observó una frecuencia mayor en el recuerdo y asociación de palabras mediante la intervención de las letras.

- Con lo dicho anteriormente podemos concluir en que se pudo comprobar la hipótesis de esta investigación.

\section{Referencias Bibliográficas}

Ayala, N, (4 de febrero del 2014). Memoria. Recuperado de: https://mundogestalt.com/memoria/

De la Mata, M,.y Sánchez J. (1996). Psicología de la Memoria. Practicas experimentales. España: Algaida editores.

Kerlinger, F,.y Lee, H. (2002). Investigación del comportamiento. Chile: McGraw-Hill.

Morris, C., y Maisto, A. (2005). Introducción a la psicología. México: Pearson Educación. 